\title{
Low Cost Design of a Hybrid Architecture of Integer Inverse DCT for H.264, VC-1, AVS, and HEVC
}

\author{
Muhammad Martuza and Khan A. Wahid \\ Department of Electrical and Computer Engineering, University of Saskatchewan, Saskatoon, SK, Canada S7N 5A9 \\ Correspondence should be addressed to Khan A. Wahid, khan.wahid@usask.ca
}

Received 2 December 2011; Accepted 6 March 2012

Academic Editor: Maurizio Martina

Copyright ( $) 2012$ M. Martuza and K. A. Wahid. This is an open access article distributed under the Creative Commons Attribution License, which permits unrestricted use, distribution, and reproduction in any medium, provided the original work is properly cited.

\begin{abstract}
The paper presents a unified hybrid architecture to compute the $8 \times 8$ integer inverse discrete cosine transform (IDCT) of multiple modern video codecs-AVS, H.264/AVC, VC-1, and HEVC (under development). Based on the symmetric structure of the matrices and the similarity in matrix operation, we develop a generalized "decompose and share" algorithm to compute the $8 \times 8$ IDCT. The algorithm is later applied to four video standards. The hardware-share approach ensures the maximum circuit reuse during the computation. The architecture is designed with only adders and shifters to reduce the hardware cost significantly. The design is implemented on FPGA and later synthesized in CMOS 0.18 um technology. The results meet the requirements of advanced video coding applications.
\end{abstract}

\section{Introduction}

In recent years, different video applications use different video standards, such as H.264/AVC [1], VC-1 [2], and AVS [3]. To improve the coding efficiency further, recently a joint collaboration team on video coding (JCT-VC) is drafting a next generation video coding standards, known tentatively as high efficient video coding (HEVC or H.265) [4]. The target bit rate is half of that of H.264/AVC. Besides, several other effective techniques are proposed in the draft to reduce the complexity of the encoder such as improved intrapicture coding, and simpler VLC coefficients [5]. As a result of these new features, experts predict that the HEVC will dominate the future multimedia market.

In order to meet up the present and future demands of different multimedia applications, it becomes necessary to develop a unified video decoder that can support all popular video standards on a single platform. In recent years, there is a growing interest to develop multistandard inverse transform architectures for advanced multimedia applications. However, most of them do not support AVS, the video codec developed by Chinese government that became the core technology of China Mobile Multimedia Broadcasting (CMMB) [6]. None of the existing works supports the HEVC; thought it is not finalized yet, considering the future prospective of the HEVC [7], it is important to start exploring possible implementation in hardware of the transform unit discussed in the draft.

In this paper, we present a new generalized algorithm and its hardwire implementation of an $8 \times 8$ IDCT architecture. The scheme is based on matrix decomposition with sparse matrices and offset computations. These sparse matrices are derived in a way that can be reused maximum number of times during decoding different inverse matrices. All multipliers in the design are replaced by adders and shifters. In the scheme, we first split the $8 \times 8$ transformation matrix into two small $4 \times 4$ matrices by applying permutation techniques. Then we concurrently perform separate operations on these two matrices to compute the output. It enables parallel operation and yields high throughput, which eventually helps meet the coding requirement of the high resolution video. 
The proposed generalized algorithm is later applied to compute the $8 \times 8$ integer IDCT of AVS. Then we identify the submatrices of AVS and reuse them to compute the IDCT of VC-1. We follow the same principle to compute the other two IDCTs of H.264 and HEVC. For HEVC, we have used the draft matrix discussed in the recent meeting [7]; since it is not yet finalized, we have developed the generalized architecture in such a way that can be easily adjusted to accommodate any changes to the final HEVC format.

\section{Previous Works}

In recent years, some multistandard inverse transform architectures have been proposed for video applications. Lee's work in [8] presents a $8 \times 8$ multistandard IDCT architecture based on delta coefficient matrices which can support VC-1, MPEG4, and H.264. It can process up to $21.9 \mathrm{fps}$ for full HD video. Kim's work in [9] describes a design following similar approach of [8] to unify the IDCT and inverse quantization (IQ) operations for those three codecs. However, the design cannot support full HD video format. Qi's work in [10] shows an efficient integrated architecture designed for multistandard inverse transforms of MPEG-2/4, H.264, and VC-1 using factor share (FS) and adder share (AS) strategies for saving circuit resource. The work achieves $100 \mathrm{MHz}$ working frequency for full $\mathrm{HD}$ video resolution, but does not support AVS. In another interesting design [11], the authors devise a common architecture by sharing adders and multipliers to perform transform and quantization of H.264, MPEG-4, and VC- 1 . The common shortcoming of all these designs discussed in [8-11] is that none of them supports the Chinese standard, AVS, nor the HEVC.

In our previous work [12], we have developed a resource shared design using delta coefficient matrices which can compute the $8 \times 8$ IDCT of VC-1, JPEG, MPEG4, H.264/AVC, and AVS. But due to complex data scheduling and the integration of JPEG (which is an image codec), the decoding capability is limited. The design supports both HD formats, but fails to comply with super resolution (WQXGA). Liu [13] introduces another design to support multiple standards where the design throughput is low $(110.8 \mathrm{MHz})$ and cannot decode HD and WQXGA video. Fan's works in $[14,15]$ are based on another efficient matrix decomposition algorithm to compute multiple transforms; however, the work is limited to only H.264 and VC-1. There are similar works in [16-18], which are also limited to these two codecs (H.264 and VC-1).

In this paper, we present a generalized low-cost algorithm and its single chip implementation to compute all four modern video standards (AVS, H.264, VC-1, and HEVC). The design meets the requirement of high performance video coding as it can process the HD video at $145 \mathrm{fps}$, the full $\mathrm{HD}$ video at $62 \mathrm{fps}$, and the WQXGA video at $32 \mathrm{fps}$. The proposed scheme can be applied to both forward and inverse transformation; however, here we only show the implementation for the inverse process (targeted for decoders).
TABLe 1: Matrix coefficients of 8-point IDCT.

\begin{tabular}{lcccc}
\hline & AVS [3] & VC-1 [2] & H.264 [1] & HEVC [7] \\
\hline$a$ & 8 & 12 & 8 & 64 \\
$b$ & 10 & 16 & 12 & 89 \\
$c$ & 9 & 15 & 10 & 75 \\
$d$ & 6 & 9 & 6 & 50 \\
$e$ & 2 & 4 & 3 & 18 \\
$f$ & 10 & 16 & 8 & 83 \\
$g$ & 4 & 6 & 4 & 36 \\
\hline
\end{tabular}

\section{Proposed Generalized Algorithm for $8 \times 8$ IDCT}

In a video compression system, the transform coding usually employs an 8-point II-type DCT. Since, the forward DCT uses the same basis coefficients and is the transpose of the IDCT matrix, the proposed IDCT scheme is easily applicable to it without any added cost or complexity. The 8-point 1D forward and inverse DCT coefficient matrices are expressed in general form as $F$ and $I$ respectively (below in (1), where, $a, b, c \ldots, g$ denote seven different transform coefficients):

$$
\begin{aligned}
F & =\left[\begin{array}{cccccccc}
a & a & a & a & a & a & a & a \\
b & c & d & e & -e & -d & -c & -b \\
f & g & -g & -f & -f & -g & g & f \\
c & -e & -b & -d & d & b & e & -c \\
a & -a & -a & a & a & -a & -a & a \\
d & -b & e & c & -c & -e & b & -d \\
g & -f & f & -g & -g & f & -f & g \\
e & -d & c & -b & b & -c & d & -e
\end{array}\right], \\
I & =\left[\begin{array}{cccccccc}
a & b & f & c & a & d & g & e \\
a & c & g & -e & -a & -b & -f & -d \\
a & d & -g & -b & -a & e & f & c \\
a & e & -f & -d & a & c & -g & -b \\
a & -e & -f & d & a & -c & -g & b \\
a & -d & -g & b & -a & -e & f & -c \\
a & -c & g & e & -a & b & -f & d \\
a & -b & f & -c & a & -d & g & -e
\end{array}\right] .
\end{aligned}
$$

In this paper, we have denoted the $8 \times 8$ IDCT transform matrices for AVS, VC-1, H.264/AVC, and HEVC by the letters $A, V, H$ and $H V$ respectively. These seven coefficients $(a, b, c \ldots, g)$ for each of the transforms are different, but integer in nature (as shown in Table 1).

3.1. Development of a Generalized "Decompose and Share" Algorithm. First of all, we derive a generalized matrix decomposition scheme by utilizing the symmetric structure of the matrices and factoring the $8 \times 8$ matrix into two $4 \times 4$ sub-matrices as shown below:

$$
I=P_{0} \cdot I_{0} \text {, }
$$


where

$$
\begin{gathered}
I_{0}=\left[\begin{array}{cccccccc}
a & 0 & f & 0 & a & 0 & g & 0 \\
a & 0 & g & 0 & -a & 0 & -f & 0 \\
a & 0 & -g & 0 & -a & 0 & f & 0 \\
a & 0 & -f & 0 & a & 0 & -g & 0 \\
0 & -e & 0 & d & 0 & -c & 0 & b \\
0 & -d & 0 & b & 0 & -e & 0 & -c \\
0 & -c & 0 & e & 0 & b & 0 & d \\
0 & -b & 0 & -c & 0 & -d & 0 & -e
\end{array}\right], \\
P_{0}=\left[\begin{array}{cccccccc}
1 & 0 & 0 & 0 & 0 & 0 & 0 & -1 \\
0 & 1 & 0 & 0 & 0 & 0 & -1 & 0 \\
0 & 0 & 1 & 0 & 0 & -1 & 0 & 0 \\
0 & 0 & 0 & 1 & -1 & 0 & 0 & 0 \\
0 & 0 & 0 & 1 & 1 & 0 & 0 & 0 \\
0 & 0 & 1 & 0 & 0 & 1 & 0 & 0 \\
0 & 1 & 0 & 0 & 0 & 0 & 1 & 0 \\
1 & 0 & 0 & 0 & 0 & 0 & 0 & 1
\end{array}\right] .
\end{gathered}
$$

The computational complexity of $P_{0}$ is only 8 additions. To reduce the complexity of $I_{0}$, we use permutation techniques by performing the operations: $I_{0}=\tilde{I} \cdot P_{C}$.

Where

$$
\begin{gathered}
P_{C}=\left[\begin{array}{cccccccc}
1 & 0 & 0 & 0 & 0 & 0 & 0 & 0 \\
0 & 0 & 1 & 0 & 0 & 0 & 0 & 0 \\
0 & 0 & 0 & 0 & 1 & 0 & 0 & 0 \\
0 & 0 & 0 & 0 & 0 & 0 & 1 & 0 \\
0 & 1 & 0 & 0 & 0 & 0 & 0 & 0 \\
0 & 0 & 0 & 1 & 0 & 0 & 0 & 0 \\
0 & 0 & 0 & 0 & 0 & 1 & 0 & 0 \\
0 & 0 & 0 & 0 & 0 & 0 & 0 & 1
\end{array}\right], \\
\widetilde{I}=\left[\begin{array}{ccccccccc}
a & f & a & g & 0 & 0 & 0 & 0 \\
a & g & -a & -f & 0 & 0 & 0 & 0 \\
a & -g & -a & f & 0 & 0 & 0 & 0 \\
a & -f & a & -g & 0 & 0 & 0 & 0 \\
0 & 0 & 0 & 0 & -e & d & -c & b \\
0 & 0 & 0 & 0 & -d & b & -e & -c \\
0 & 0 & 0 & 0 & -c & e & b & d \\
0 & 0 & 0 & 0 & -b & -c & -d & -e
\end{array}\right] .
\end{gathered}
$$

There is no computational cost for $P_{C}$ as it only permutes the input data set (just needs rewiring). $\tilde{I}$ can be further decomposed into two $4 \times 4$ submatrices, $\widetilde{I}_{00}$ and $\widetilde{I}_{11}$, by the direct sum operation ( $\oplus$ ") as shown below:

$$
\widetilde{I}=\widetilde{I}_{00} \oplus \widetilde{I}_{11}
$$

Thus,

$$
I=P_{0} \cdot\left(\tilde{I}_{00} \oplus \tilde{I}_{11}\right) \cdot P_{C}
$$

where

$$
\begin{gathered}
\tilde{I}_{00}=\left[\begin{array}{cccc}
a & f & a & g \\
a & g & -a & -f \\
a & -g & -a & f \\
a & -f & a & -g
\end{array}\right], \\
\tilde{I}_{11}=\left[\begin{array}{cccc}
-e & d & -c & b \\
-d & b & -e & -c \\
-c & e & b & d \\
-b & -c & -d & -e
\end{array}\right] .
\end{gathered}
$$

Equation (6) forms the general expression of (1). We will use $\widetilde{I}_{00}$ and $\widetilde{I}_{11}$ as the basic building blocks to compute other $8 \times 8$ IDCTs. Since, the coefficients in $\widetilde{I}_{00}$ and $\widetilde{I}_{11}$ are fixed, they can be independently implemented, enabling fast computation.

In the following section, we show how (6) can be applied to different IDCT matrices. Another new feature of the proposed scheme is that we take the advantage of the similarity in matrix operation to further optimize the implementation. First of all, we apply (6) to efficiently implement the transformation matrix of AVS. Based on it and the generalized structure, we develop the matrix of VC-1 so that we can share as many units (from AVS) as possible. Next, we develop the IDCT matrix of H.264 based on the same principle (decompose and share from AVS and VC-1). In this stage, we are able to achieve the maximum sharing as it will be shown later (in Section 3.4) that the implementation of H.264 does not cost any extra hardware. Finally, we develop the IDCT of HEVC by further decomposing and reusing the units already implemented (with a minimum addition of extra units).

3.2. Matrix Decomposition for AVS. Let us now construct $A$ (from (1) and Table 1) and apply (6) to compute the $4 \times 4$ submatrices, $\widetilde{A}_{00}$ and $\widetilde{A}_{11}$. We then right shift $\widetilde{A}_{00}$ by three bits and decompose it as follows:

$$
\frac{\widetilde{A}_{00}}{8}=\left[\begin{array}{cccc}
1 & \frac{5}{4} & 1 & \frac{1}{2} \\
1 & \frac{1}{2} & -1 & -\frac{5}{4} \\
1 & -\frac{1}{2} & -1 & \frac{5}{4} \\
1 & -\frac{5}{4} & 1 & -\frac{1}{2}
\end{array}\right]=A_{1} \cdot A_{2},
$$

where

$$
A_{1}=\left[\begin{array}{cccc}
1 & 0 & 1 & 0 \\
0 & 1 & 0 & 1 \\
0 & 1 & 0 & -1 \\
1 & 0 & -1 & 0
\end{array}\right],
$$

$$
A_{2}=\left[\begin{array}{cccc}
1 & 0 & 1 & 0 \\
1 & 0 & -1 & 0 \\
0 & \frac{5}{4} & 0 & \frac{1}{2} \\
0 & \frac{1}{2} & 0 & -\frac{5}{4}
\end{array}\right] .
$$

Like $P_{0}$, the computational cost of $A_{1}$ is only 4 additions. For $A_{2}$, we implement $(5 / 4) \cdot x$ as $(1+1 / 4) \cdot x$-that is right 
shift $x$ (arbitrary data) by two bits and then add with $x$. So, the cost is 6 add and 6 shift operations. Thus in (8), the total computational cost is 10 addition and 6 shift operations. In similar way, we can decompose $\widetilde{A}_{11}$ as shown below:

$$
\frac{\tilde{A}_{11}}{4}=\left[\begin{array}{rccc}
-\frac{1}{2} & \frac{3}{2} & -\frac{9}{4} & \frac{5}{2} \\
-\frac{3}{2} & \frac{5}{2} & -\frac{1}{2} & -\frac{9}{4} \\
-\frac{9}{4} & \frac{1}{2} & \frac{5}{2} & \frac{3}{2} \\
-\frac{5}{2} & -\frac{9}{4} & -\frac{3}{2} & -\frac{1}{2}
\end{array}\right]=A_{3} \cdot A_{4}
$$

where

$$
A_{3}=\left[\begin{array}{cccc}
-1 & 0 & \frac{3}{2} & 1 \\
0 & 1 & 1 & -\frac{3}{2} \\
-\frac{3}{2} & 1 & -1 & 0 \\
-1 & -\frac{3}{2} & 0 & -1
\end{array}\right], \quad A_{4}=\left[\begin{array}{cccc}
\frac{3}{2} & 0 & 0 & -1 \\
0 & \frac{3}{2} & 1 & 0 \\
0 & 1 & -\frac{3}{2} & 0 \\
1 & 0 & 0 & \frac{3}{2}
\end{array}\right] .
$$

For both $A_{3}$ and $A_{4}$, the coefficient (3/2) can be shared and the cost is: 12 additions and 4 shift operations for $A_{3} ; 8$ additions and 4 shift operations for $A_{4}$. From (8)-(10), we can summarize the final expression of the $8 \times 8$ IDCT for AVS as:

$$
A=4 \cdot P_{0} \cdot\left[\left(A_{1} \cdot 2 A_{2}\right) \oplus\left(A_{3} \cdot A_{4}\right)\right] \cdot P_{C} .
$$

Thus, the total computational cost to implement $A$ is 38 additions and 26 shift operations. In the next section, we will apply (6) to VC-1 and subsequently decompose the matrix in a way so that we can reuse the units already developed for the AVS (from (12)).

3.3. Matrix Decomposition for VC-1. We follow the same principles, as discussed in (8) and (10), to decompose the $8 \times 8$ IDCT for the VC- 1 :

$$
V=P_{0} \cdot \tilde{V} \cdot P_{C}
$$

where

$$
\tilde{V}=\tilde{V}_{00} \oplus \tilde{V}_{11} .
$$

Now considering the symmetric property and the coefficient distribution patterns between $\widetilde{A}_{00} / 8$ (in (8)) and $\widetilde{V}_{00} / 8$, we decompose $\tilde{V}_{00} / 8$ as:

$$
\frac{\tilde{V}_{00}}{8}=\left[\begin{array}{cccc}
\frac{3}{2} & 2 & \frac{3}{2} & \frac{3}{4} \\
\frac{3}{2} & \frac{3}{4} & -\frac{3}{2} & -2 \\
\frac{3}{2} & -\frac{3}{4} & -\frac{3}{2} & 2 \\
\frac{3}{2} & -2 & \frac{3}{2} & -\frac{3}{4}
\end{array}\right]=A_{1} \cdot V_{2},
$$

where

$$
\begin{aligned}
& V_{2}=\left[\begin{array}{cccc}
\frac{3}{2} & 0 & \frac{3}{2} & 0 \\
\frac{3}{2} & 0 & -\frac{3}{2} & 0 \\
0 & 2 & 0 & \frac{3}{4} \\
0 & \frac{3}{4} & 0 & -2
\end{array}\right]=2 A_{2}-V_{3}, \\
& V_{3}=\left[\begin{array}{cccc}
\frac{1}{2} & 0 & \frac{1}{2} & 0 \\
\frac{1}{2} & 0 & -\frac{1}{2} & 0 \\
0 & \frac{1}{2} & 0 & \frac{1}{4} \\
0 & \frac{1}{4} & 0 & -\frac{1}{2}
\end{array}\right] .
\end{aligned}
$$

From (16), (15) can be reexpressed as:

$$
\frac{\tilde{V}_{00}}{8}=A_{1} \cdot\left(2 A_{2}-V_{3}\right) .
$$

Now it can be seen how the implementation of AVS matrix (from (12)) can be reused in (17). This matrix decomposition enables hardware sharing and results in significant saving in implementation resources. From (17), the total cost of $V_{3}$ and $\tilde{V}_{00} / 8$ is 8 additions and 6 shift operations.

Next based on our careful observation between the computational similarities between $\widetilde{A}_{11} / 4$ (in (10)) and $\widetilde{V}_{11} / 8$, we devise the decomposition scheme of $\widetilde{V}_{11} / 8$ as:

$$
\frac{\tilde{V}_{11}}{8}=\left[\begin{array}{cccc}
-\frac{1}{2} & \frac{9}{8} & -\frac{15}{8} & 2 \\
-\frac{9}{8} & 2 & -\frac{1}{2} & -\frac{15}{8} \\
-\frac{15}{8} & \frac{1}{2} & 2 & \frac{9}{8} \\
-2 & -\frac{15}{8} & -\frac{9}{8} & -\frac{1}{2}
\end{array}\right]=V_{4} \cdot A_{4 v}
$$

where $V_{4}=A_{3}+A_{3 V}$,

$$
A_{4 v}=\left[\begin{array}{cccc}
\frac{1}{4} & 0 & 0 & -1 \\
0 & \frac{1}{4} & 1 & 0 \\
0 & 1 & -\frac{1}{4} & 0 \\
1 & 0 & 0 & \frac{1}{4}
\end{array}\right], \quad A_{3 v}=\left[\begin{array}{cccc}
-1 & -\frac{3}{2} & 0 & -1 \\
\frac{3}{2} & -1 & 1 & 0 \\
0 & 1 & 1 & -\frac{3}{2} \\
1 & 0 & -\frac{3}{2} & -1
\end{array}\right] \text {. }
$$

By substituting (19) in (18), $\widetilde{V}_{11} / 8$ is expressed as:

$$
\frac{\tilde{V}_{11}}{8}=\left(A_{3}+A_{3 V}\right) \cdot A_{4 v} \text {. }
$$


Note that $A_{4 v}$ in (19) is structurally similar to $A_{4}$ in (10) except the change in the diagonal coefficients. So we only need to implement it; the rest is shared from the architecture of $A_{4}$. We do so by adding 4 multiplexers at the output of the four left diagonal elements of $A_{4}$ matrix. Then according to (19), we reuse $A_{3}$ to compute $V_{4}$. As the new matrix $A_{3 v}$ can be derived from $A_{3}$ by rearranging the rows and changing the polarity of some input bits, we share it from the design of $A_{3}$ by adding 4 multiplexers only. Finally, the expression of $\widetilde{V}_{00}$ and $\tilde{V}_{11}$ from (17) and (20) are substituted in (13) to get the final expression of the IDCT for VC-1:

$$
V=8 \cdot P_{0} \cdot\left\{\left[A_{1} \cdot\left(2 A_{2}-V_{3}\right)\right] \oplus\left[\left(A_{3}+A_{3 V}\right) \cdot A_{4 v}\right]\right\} \cdot P_{C} .
$$

It is seen from (21) that to implement $V$, the only new unit that is required is $V_{3}$; the rest is shared from the implementation of AVS (from (12)). So, the total computational cost for $\mathrm{VC}-1$ is 12 additions and 10 shift operations.

3.4. Matrix Decomposition for H.264/AVC. Following similar procedure illustrated in the two previous sections, we can simplify the $8 \times 8$ transformation matrix for H.264/AVC as shown below:

$$
H=P_{0} \cdot \tilde{H} \cdot P_{C},
$$

where

$$
\widetilde{H}=\widetilde{H}_{00} \oplus \widetilde{H}_{11} .
$$

In order to ensure the maximum unit sharing, we decompose $\widetilde{H}_{00} / 8$ as below:

$$
\frac{\tilde{H}_{00}}{8}=\left[\begin{array}{cccc}
1 & 1 & 1 & \frac{1}{2} \\
1 & \frac{1}{2} & -1 & -1 \\
1 & -\frac{1}{2} & -1 & 1 \\
1 & -1 & 1 & -\frac{1}{2}
\end{array}\right]=A_{1} \cdot A_{2 h}
$$

where

$$
A_{2 h}=\left[\begin{array}{cccc}
1 & 0 & 1 & 0 \\
1 & 0 & -1 & 0 \\
0 & 1 & 0 & \frac{1}{2} \\
0 & \frac{1}{2} & 0 & -1
\end{array}\right] .
$$

In (24), $A_{1}$ is directly reused from (12). To share $A_{2 h}$ from the architecture of $A_{2}$ we simply add two multiplexer units.
So there is no additional cost in terms of adders and shifters to compute $\widetilde{H}_{00} / 8$. Similarly, we can decompose $\widetilde{H}_{11} / 8$ as:

$$
\frac{\tilde{H}_{11}}{8}=\left[\begin{array}{rrrc}
-\frac{3}{8} & \frac{3}{4} & -\frac{5}{4} & \frac{3}{2} \\
-\frac{3}{4} & \frac{3}{2} & -\frac{3}{8} & -\frac{5}{4} \\
-\frac{5}{4} & \frac{3}{8} & \frac{3}{2} & \frac{3}{4} \\
-\frac{3}{2} & -\frac{5}{4} & -\frac{3}{4} & -\frac{3}{8}
\end{array}\right]=A_{3 h} \cdot A_{4 v}
$$

where

$$
A_{3 h}=\left[\begin{array}{cccc}
-\frac{3}{2} & -1 & 1 & 0 \\
1 & 0 & \frac{3}{2} & -1 \\
-1 & \frac{3}{2} & 0 & -1 \\
0 & -1 & -1 & -\frac{3}{2}
\end{array}\right] \text {. }
$$

Here $A_{4 v}$ is directly reused from (21) and we share $A_{3 h}$ from the architecture of $A_{3}$. In this sharing we do not even need to use any multiplexers, because we have already done so while sharing $A_{3 v}$ from $A_{3}$ in Section 3.3. The final expression of the $8 \times 8$ IDCT for H.264 (with all shared units) can be summarized as follows:

$$
H=8 \cdot P_{0} \cdot\left\{\left[A_{1} \cdot A_{2 h}\right] \oplus\left[A_{3 h} \cdot A_{4 v}\right]\right\} \cdot P_{C} .
$$

It is interesting to note that all terms in (28) are implemented from the terms of (12) and (21); thus, in the proposed scheme, there is no additional cost to implement the IDCT for H.264 which results in significant hardware savings.

3.5. Matrix Decomposition for HEVC. In this section, we develop the transformation matrix for the HEVC based on the principles described before. The $8 \times 8$ matrix can be decomposed as:

$$
H V=P_{0} \cdot \widetilde{H V} \cdot P_{C}
$$


where

$$
\begin{gathered}
\widetilde{H V}=\widetilde{H V_{00}} \oplus \widetilde{H V_{11}}, \\
\frac{\widetilde{H V_{00}}}{4}=\left[\begin{array}{cccc}
16 & \frac{83}{4} & 16 & 9 \\
16 & 9 & -16 & -\frac{83}{4} \\
16 & -9 & -16 & \frac{83}{4} \\
16 & -\frac{83}{4} & 16 & -9
\end{array}\right]=A_{1} \cdot\left(16 A_{2}+H V_{1}\right),
\end{gathered}
$$$$
H V_{1}=\left[\begin{array}{cccc}
0 & 0 & 0 & 0 \\
0 & 0 & 0 & 0 \\
0 & \frac{3}{4} & 0 & 1 \\
0 & 1 & 0 & -\frac{3}{4}
\end{array}\right]
$$

The computational cost of $H V_{1}$ is 4 additions and 4 shift operations. Here the coefficient $(3 / 4) \cdot x$ is factorized as $(x-x / 4)$. So the cost of $\widetilde{H V_{00}}$ in $(30)$ is 4 additions and 8 shift operations. Similarly, we decompose $\widetilde{H V_{11}} / 4$ as:

$$
\widetilde{H V_{11}} \frac{\widetilde{1}}{4}=\left[\begin{array}{cccc}
-\frac{9}{2} & \frac{25}{2} & -\frac{75}{4} & \frac{89}{4} \\
-\frac{25}{2} & \frac{89}{4} & -\frac{9}{2} & -\frac{75}{4} \\
-\frac{75}{4} & \frac{9}{2} & \frac{89}{4} & \frac{25}{2} \\
-\frac{89}{4} & -\frac{75}{4} & -\frac{25}{2} & -\frac{9}{2}
\end{array}\right]=\left(8 A_{3}+H V_{2}\right) \cdot A_{4 H V},
$$

where

$$
H V_{2}=\left[\begin{array}{cccc}
\frac{7}{4} & \frac{5}{4} & -2 & 0 \\
-\frac{5}{4} & 0 & -\frac{7}{4} & 2 \\
2 & -\frac{7}{4} & 0 & \frac{5}{4} \\
0 & 2 & \frac{5}{4} & \frac{7}{4}
\end{array}\right], \quad A_{4 H V}=\left[\begin{array}{cccc}
2 & 0 & 0 & -1 \\
0 & 2 & 1 & 0 \\
0 & 1 & -2 & 0 \\
1 & 0 & 0 & 2
\end{array}\right]
$$

Combining (29)-(32), we compute the proposed $8 \times 8$ IDCT for HEVC as given below:

$$
\begin{aligned}
& H V=4 \cdot P_{0} \cdot\{ {\left[A_{1} \cdot\left(16 A_{2}+H V_{1}\right)\right] } \\
&\left.\oplus\left[\left(8 A_{3}+H V_{2}\right) \cdot A_{4 H V}\right]\right\} \cdot P_{C} .
\end{aligned}
$$

In (34), only the new matrices, $H V_{1}$ and $H V_{2}$, will be implemented and the rest will be shared from (12). So the

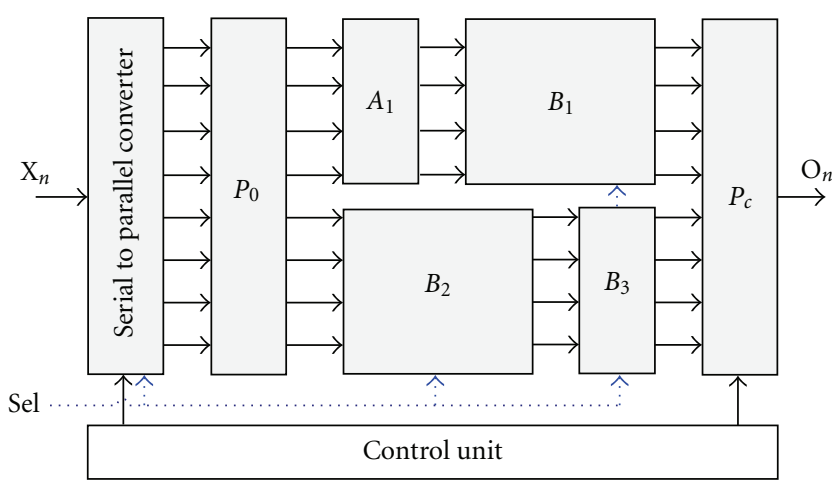

FIgURE 1: Block diagram of the proposed architecture.

total computational cost to implement $H V$ in the proposed design is 24 additions and 28 shift operations. It is important to note that we have carefully decomposed $H V$ so that if there is any change in the final standard, all one needs to do is to update (30) and (32) with new parameters without interrupting the entire design. In summery, the proposed unified design costs 74 additions and 64 shift operations to perform the inverse transformation of four defined video standards.

\section{Hardware Implementation of the Shared Architecture}

In the implementation of the multistandard architectures on a single platform, we have shared the entire hardware unit of the $4 \times 4$ matrices, instead of sharing individual adders, shifters, or other factors (as done in [10]). It ensures maximum reduction of hardware cost in our design. The overall block diagram of our proposed scheme is shown in Figure 1 . We can see from Figure 1 that the $P_{0}$ block splits the 8 -point decomposition process to two independent 4-point processes; since these two processes work concurrently, the design throughput is highly increased. The blocks $B_{1}, B_{2}$, and $B_{3}$ perform different operations (shared) as shown in Table 2 .

Figure 2(a) shows the design of the serial to parallel converter (S2P) block. It performs left shift and then stores the input one by one into eight registers in 8 clock cycles, and at the 9th cycle, all stored input samples are sent to next block, $P_{0}$. Here the S2P block apparently functions like a temporary memory buffer as it stores the rows of the input matrix inside eight registers. As a result, the proposed design does not require additional memory architecture. The wrapper architecture $\left(P_{C}\right)$ is shown in Figure 2(b). In this multicodec system, only one IDCT and its associated computational units are activated at a time by the control unit and the select pin (Sel); the rest is disabled. The other blocks are shown in Figure 3. In different stages of the design, several multiplexers are used to ensure proper computation of the IDCT in operation. Finally, the $P_{C}$ block combines two different set of data and generates one output. In Figure $3, \mathrm{In}_{0}, \mathrm{In}_{1}, \ldots, \mathrm{In}_{3}$ represent the inputs coming from the previous block and $\mathrm{Out}_{0}, \mathrm{Out}_{1}, \ldots, \mathrm{Out}_{3}$ represent the outputs going to the next block. As an example, in 
TABLE 2: Controlling selection of subblocks.

\begin{tabular}{llccc}
\hline Select (Sel) & IDCT & $B_{1}$ & $B_{2}$ & $B_{3}$ \\
& & $(16 / 2) \cdot\left(A_{2} / A_{2 h}\right)-\left(V_{3} / H V_{1}\right)$ & $(8 / 1) \cdot\left[A_{3} / A_{3 h} /\left(A_{3}+A_{3 v}\right)\right]+H V_{2}$ & $A_{4} / A_{4 v} / A_{4 H V}$ \\
\hline 00 & AVS & $2 A_{2}$ & $A_{3}$ & $A_{4}$ \\
01 & VC-1 & $2 A_{2}-V_{3}$ & $\left(A_{3}+A_{3 v}\right)$ & $A_{4 v}$ \\
10 & H.264 & $A_{2 h}$ & $A_{3 h}$ & $A_{4 v}$ \\
11 & HEVC & $16 A_{2}-H V_{1}$ & $8 A_{3}+H V_{2}$ & $A_{4 H V}$ \\
\hline
\end{tabular}

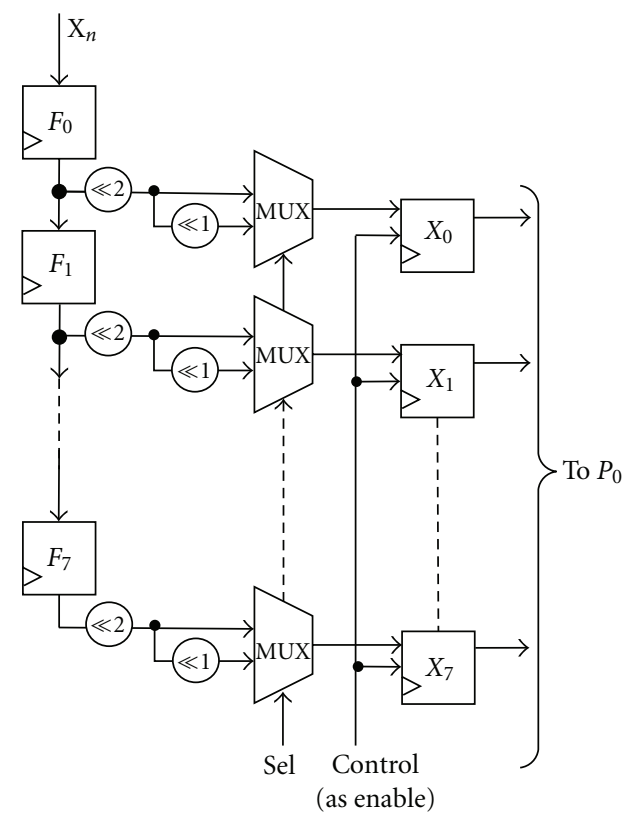

(a)

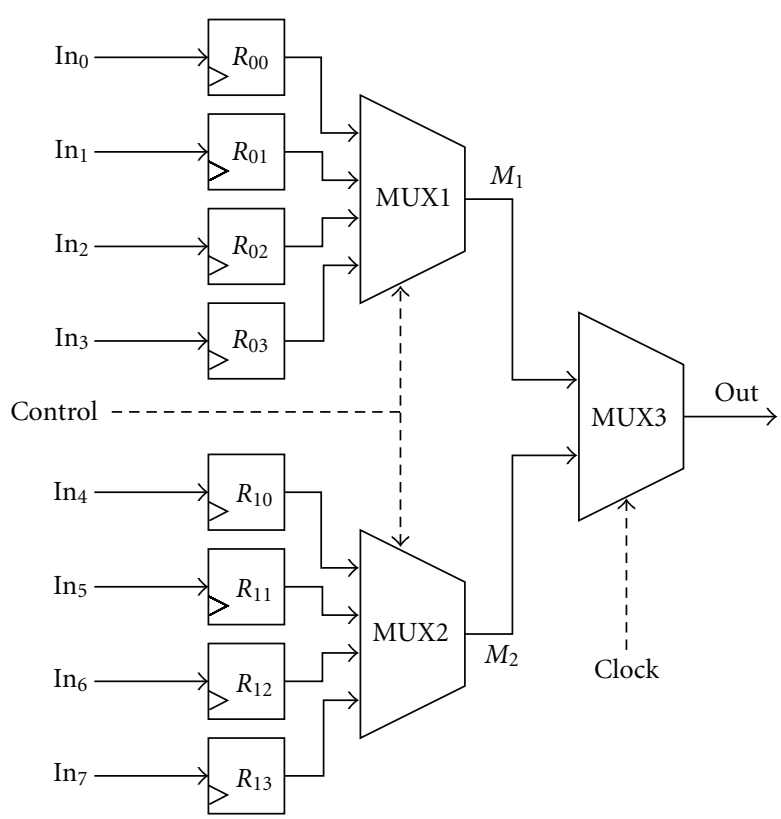

(b)

FIgURe 2: (a) Serial to parallel converter with shifting; (b) output wrapper ( $P_{C}$ block).

TABLE 3: Comparison of the cost of adders and shifters.

\begin{tabular}{lcc}
\hline Codecs & $\begin{array}{c}\text { Number } \\
\text { of adders }\end{array}$ & $\begin{array}{c}\text { Number of } \\
\text { shifters }\end{array}$ \\
\hline JPEG + MPEG-2/4 + H.264 + VC-1 in [8] & 112 & - \\
JPEG + MPEG-2/4 + H.264 + VC-1 in [10] & 70 & - \\
H.264 + VC-1 in [14] & 76 & 28 \\
MPEG-2/4 + H.264 + VC-1 + AVS in [13] & 76 & - \\
JPEG + MPEG-2/4 + H.264 + VC-1 + AVS & 58 & 31 \\
in [12] & & \\
\hline Proposed-H.264 + VC-1 + AVS + HEVC & 74 & 64 \\
\hline
\end{tabular}

Figure 3(c) for the shared design of $V_{3} / H V_{1}$, the inputs are coming from $A_{2} / A_{2 h}$ subblock and the outputs are going to $P_{C}$ block.

The state diagram of the control unit is shown in Figure 4. Here, " $r$ " is reset and " $c$ " is a 3-bit internal counter run by the system clock. There are one reset and four active states. The states of the control signals are also shown in the diagram; for example, in state 1 (S1), S2P is storing the input vector while the output wrapper $\left(P_{C}\right.$ block) enables $R_{00}$ from MUX1 and $R_{10}$ from MUX2. Table 2 shows the units that are active depending on the status of the select pin. For example, the select signal will be " 00 " when the user wants to perform the IDCT of AVS codec. In that case, $B_{1}, B_{2}$, and $B_{3}$ will function as $2 \cdot A_{2}, A_{3}$, and $A_{4}$, respectively (the rest is inactive as found in (12)).

\section{Performance Analysis and Comparisons}

The proposed design is implemented in Verilog and its operation is verified using Xilinx Vertex4 LX60 FPGA. The total number of LUTs needed for this proposed architecture is 2,242 . The design is later synthesized using $0.18 \mu \mathrm{m} \mathrm{CMOS}$ technology. The architecture costs $39.3 \mathrm{~K}$ gates and $12.15 \mathrm{~K}$ standard cells with a maximum operating frequency of 200.8 MHz. The estimated power consumption is $29.9 \mathrm{~mW}$ with $3 \mathrm{~V}$ supply.

In order to demonstrate the sharing efficiency, we have compared the adder count of our design with the 8-point standalone IDCT matrices of three standards: AVS, VC1, and H.264/AVC (as presented in [12]). The results are shown in Figure 5. As of today, there is no implementation of the $8 \times 8$ IDCT of HEVC; thus, we have implemented it separately for the sake of better comparison. Now, we 


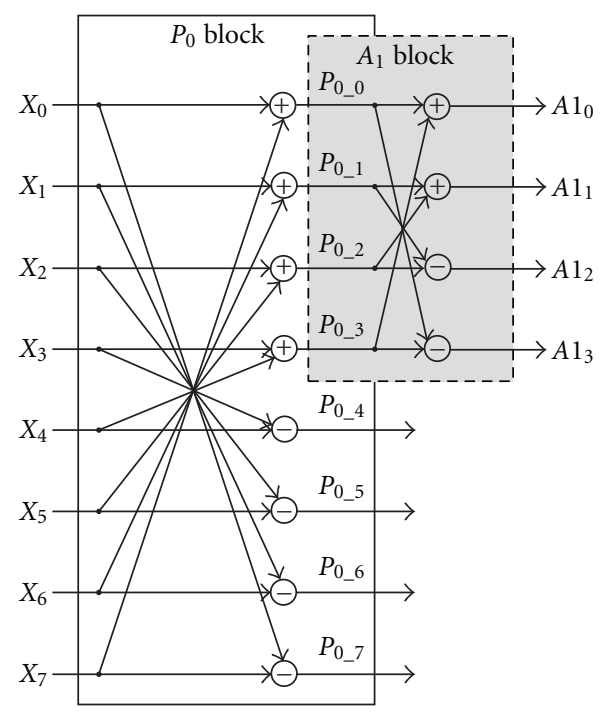

(a)

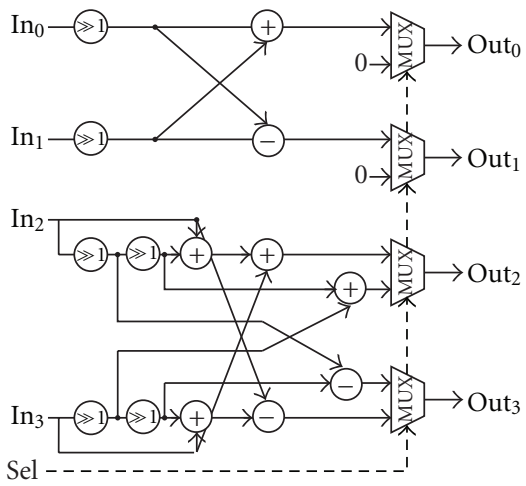

(c)

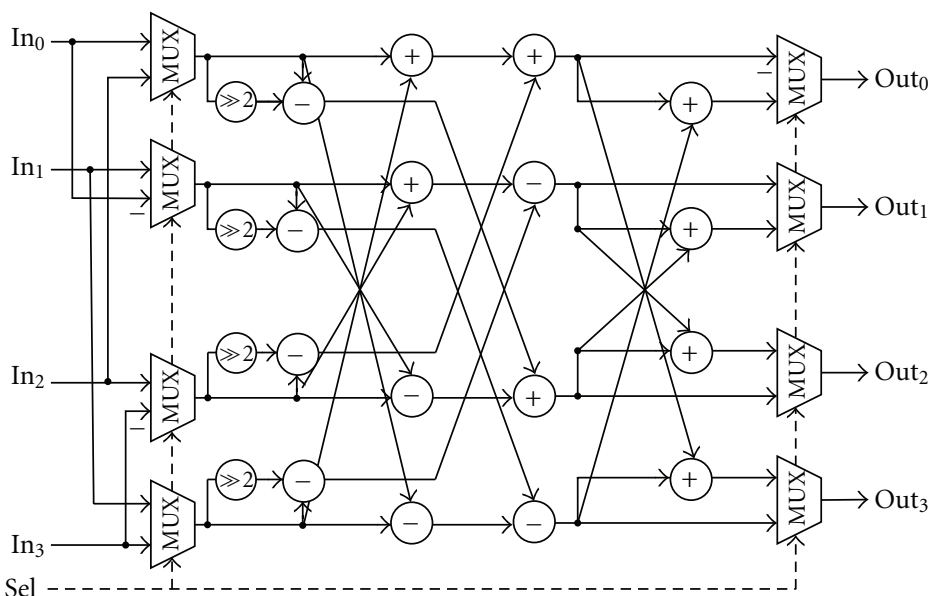

(b)
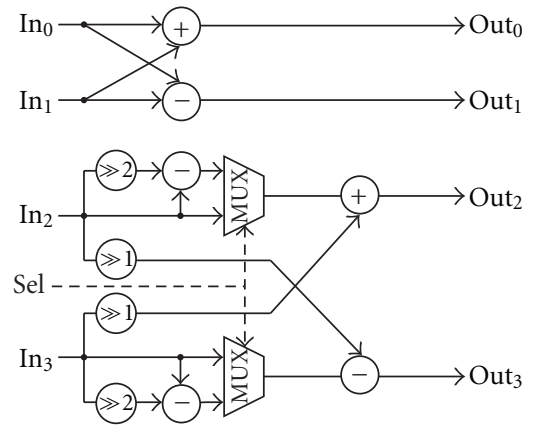

(d)

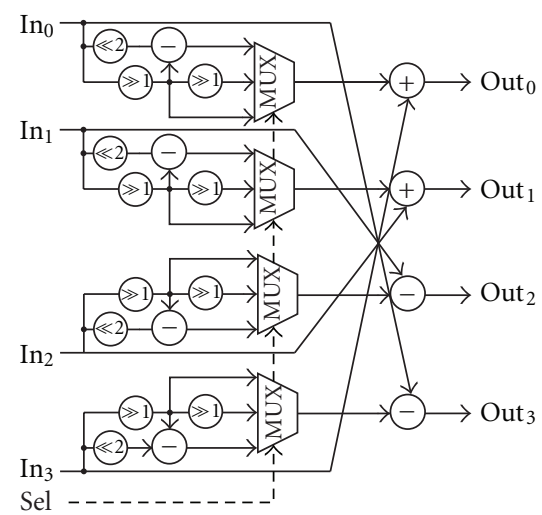

(e)

Figure 3: Shared architecture of (a) $P_{0}$ and $A_{1}$; (b) $A_{3}, A_{3 h}$ and $\left(A_{3}+A_{3 v}\right)$; (c) $V_{3}$ and $H V_{1}$; (d) $A_{2}$ and $A_{2 h}$; (e) $A_{4}, A_{4 v}$ and $A_{4 H V}$.

TABLE 4: Comparison of the resource-shared 8-point 1-D IDCT architecture.

\begin{tabular}{|c|c|c|c|c|c|c|c|c|c|}
\hline \multirow{2}{*}{ Scheme } & \multirow[b]{2}{*}{ Tech. } & \multirow[b]{2}{*}{ Gate count } & \multirow[b]{2}{*}{ Freq. (MHz) } & \multirow[b]{2}{*}{ Full HD support? } & \multirow[b]{2}{*}{ Super Resolution support? } & \multicolumn{4}{|c|}{ Supporting standards } \\
\hline & & & & & & H.264 & VC-1 & AVS & HEVC \\
\hline Lee's [8] & $0.13 \mu \mathrm{m}$ & $19.1 \mathrm{~K}$ & 136 & $\mathrm{Y}$ & o & $\mathrm{Y}$ & $\mathrm{Y}$ & o & o \\
\hline Kim's [9] & $0.13 \mu \mathrm{m}$ & $30.9 \mathrm{~K}$ & 151 & o & o & $\mathrm{Y}$ & $\mathrm{Y}$ & o & o \\
\hline Qi’s [10] & $0.13 \mu \mathrm{m}$ & $18 \mathrm{~K}$ & 100 & $\mathrm{Y}$ & o & $\mathrm{Y}$ & Y & o & o \\
\hline Lee’s [11] & $0.13 \mu \mathrm{m}$ & $10.5 \mathrm{~K}$ & 123 & o & o & $\mathrm{Y}$ & $\mathrm{Y}$ & o & o \\
\hline Wahid's [12] & $0.18 \mu \mathrm{m}$ & $19.8 \mathrm{~K}$ & 194.7 & $\mathrm{Y}$ & o & $\mathrm{Y}$ & $\mathrm{Y}$ & $\mathrm{Y}$ & o \\
\hline Liu’s [13] & $0.13 \mu \mathrm{m}$ & $16.5 \mathrm{~K}$ & 110.8 & - & - & $\mathrm{Y}$ & Y & $\mathrm{Y}$ & o \\
\hline Fan's [14] & $0.18 \mu \mathrm{m}$ & $7.14 \mathrm{~K}$ & 100 & - & - & $\mathrm{Y}$ & $\mathrm{Y}$ & o & o \\
\hline Li’s [19] & $0.18 \mu \mathrm{m}$ & $13.7 \mathrm{~K}$ & 200 & $\mathrm{Y}$ & $\mathrm{o}$ & $\mathrm{Y}$ & $\mathrm{o}$ & $\mathrm{o}$ & $\mathrm{o}$ \\
\hline Proposed & $0.18 \mu \mathrm{m}$ & $39.3 \mathrm{~K}$ & 200.8 & $\mathrm{Y}$ & $\mathrm{Y}$ & $\mathrm{Y}$ & $\mathrm{Y}$ & $\mathrm{Y}$ & $\mathrm{Y}$ \\
\hline
\end{tabular}

"Y”: yes; "o": No; “-”": no information.

can see from Figure 5 that a total of 104 adders is required to implement these four transforms without sharing. The proposed shared design can compute all of them with $28.9 \%$ less adders. Moreover, the savings achieved in individual standards due to the sharing are also marked on the figure.
It is important to note that, though the proposed design costs 38 adders to implement AVS, it does not cost any additional adder units to implement H.264. Hence, AVS and H.264 combined together cost only 38 adders (compared to 48 for standalone implementations). The cost 


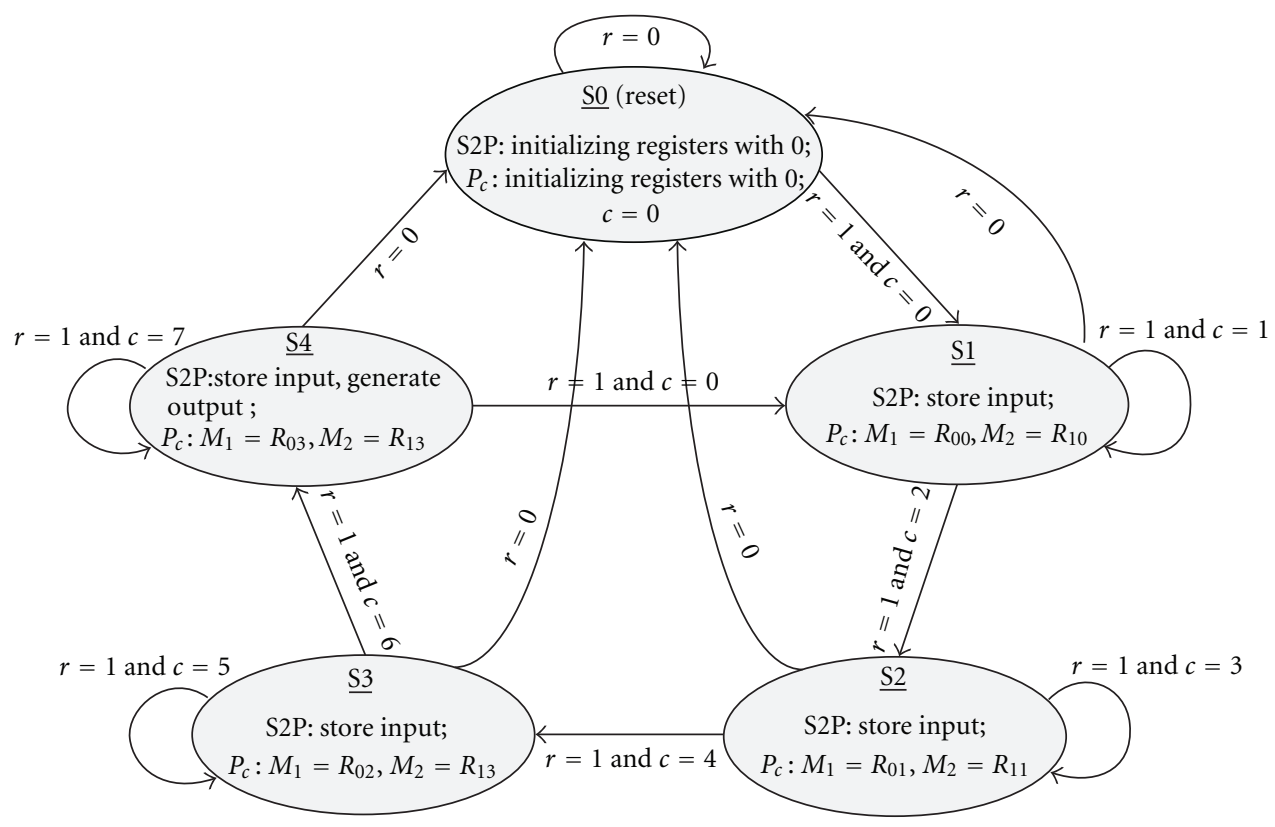

FIGURE 4: State diagram of control unit.

TABle 5: Comparison of decoding capability (minimum support of three codecs).

\begin{tabular}{|c|c|c|c|c|c|c|}
\hline \multirow{3}{*}{ Scheme } & \multicolumn{4}{|c|}{ HD resolution } & \multirow{2}{*}{\multicolumn{2}{|c|}{$\begin{array}{l}\text { Super resolution } \\
2560 \times 1600\end{array}$}} \\
\hline & \multicolumn{2}{|c|}{$1920 \times 1080$} & \multicolumn{2}{|c|}{$1280 \times 720$} & & \\
\hline & $\begin{array}{l}\text { Time to transmit } 1 \\
\text { frame }(\mathrm{msec})\end{array}$ & $\begin{array}{c}\text { Frame per } \\
\text { second (fps) }\end{array}$ & $\begin{array}{l}\text { Time to transmit } 1 \\
\text { frame }(\mathrm{msec})\end{array}$ & $\begin{array}{c}\text { Frame per } \\
\text { second (fps) }\end{array}$ & $\begin{array}{l}\text { Time to transmit } 1 \\
\text { frame }(\mathrm{msec})\end{array}$ & $\begin{array}{c}\text { Frame per } \\
\text { second (fps) }\end{array}$ \\
\hline Lee's [8] & 22.8 & 44 & 10.2 & 98 & $\mathrm{x}$ & $\mathrm{x}$ \\
\hline Kim’s[9] & $\mathrm{x}$ & $\mathrm{x}$ & 10.2 & 98 & $\mathrm{x}$ & $\mathrm{x}$ \\
\hline Qi's [10] & 31.1 & 32 & 13.8 & 72 & $\mathrm{x}$ & $\mathrm{x}$ \\
\hline Wahid's [12] & 16.7 & 60 & 7.1 & 140 & $\mathrm{x}$ & $\mathrm{x}$ \\
\hline Proposed & 15.5 & 64 & 6.9 & 145 & 30.6 & 32 \\
\hline
\end{tabular}

"_": no information; "x": not supported by the hardware.

of implementing shift operation is considered insignificant in the computation. In Table 3, we compare the cost of the proposed scheme with available existing designs in the literature. None of the designs in this table supports HEVC (which is computationally expensive due to large matrix parameters as shown in Table 1). Although, the designs in $[10,12]$ cost fewer adders, it is shown later that the proposed scheme outperforms it in decoding capacity. Considering the fact that, the proposed architecture can decode the IDCT for four video codecs, it consumes the least number of adders compared to others.

In Table 4, we have summarized the performance in terms of gate count, maximum working frequency, and standard support with other designs. Only the design in [19] has frequency closer to us, but it supports only H.264. Similarly, designs in $[11,14]$ support only two codecs and accordingly cost lesser hardware than ours. Among other designs $[8-10,12,13]$ are comparable to our design as they support as many as three codecs. While working at maximum capacity, the proposed design can process 200.8 million pixels/sec.

In order to have a better assessment among comparable designs (e.g., minimum support of three codecs), in Table 5 we compare the decoding capability (using 4:2:0 lumachroma sampling) of the proposed approach with that of [8$10,12]$. In our work, the maximum achieved frame rate of a $1080 \mathrm{p}$ video is $=200.8 \times 10^{6} /(1920 \times 1080+2 \times 960$ $\times 540)=64.56 \approx 64 \mathrm{fps}$, which is the highest compared to all other designs in Table 5. Considering the current trends to use super resolution monitors, in this table we have also compared the decoding capabilities for the Wide Quad eXtended Graphics Array (WQXGA, with resolution of 2560 $\times 1600$ pixels). Thus, it can be seen that the proposed design cannot only decode AVS, H.264/AVC, VC-1, and HEVC videos, but also can maintain relatively higher operational frequency to meet the requirements of real time transmission (the target fps to transmit HD, full HD, and QWXGA video are 120, 60, and 30, resp.). From the performance analysis, 


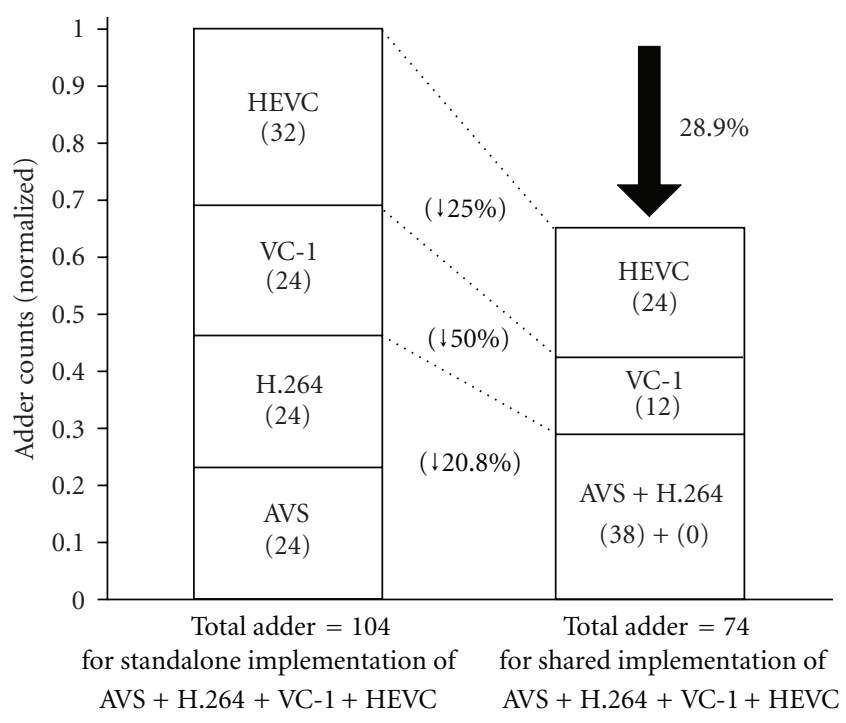

Figure 5: Cost of the proposed scheme-standalone versus costshared.

the scheme is found to be competitive as it can transmit the highest number of frames per seconds and, hence, takes the least time to transmit one frame at a given resolution.

\section{Conclusion}

In this paper, we present a generalized algorithm and a hardware-shared architecture by using the symmetric property of the integer matrices and the matrix decomposition to compute the 8-point 1-D IDCT for four modern video codecs: H.264/AVC, VC-1, AVS, and HEVC (draft in stage). The architecture is designed in such a way that can accommodate any change in the final release of the HEVC. We first apply the generalized scheme to AVS-based transform unit, and then gradually build the rest of the transform units on top of another to maximize the sharing. The performance analysis shows that the proposed design satisfies the requirement of all four codecs and achieves the highest decoding capability. Overall, the architecture is suitable for low-cost implementation in modern multicodec systems.

\section{Acknowledgment}

The authors would like to acknowledge the Natural Science and Engineering Research Council of Canada (NSERC) for its support to this research paper.

\section{References}

[1] ITU-T Rec, "H.264/ISO/IEC 14496-10 AVC," 2003.

[2] "Standard for Television: VC-1 Compressed Video Bitstream Format and Decoding Process," SMPTE 421M, 2006.

[3] GB/T 20090.1, "Information technology - Advanced coding of audio and video - Part 1: System," Chinese AVS standard.

[4] G. J. Sullivan and J.-R. Ohm, "Recent developments in standardization of high efficiency video coding (HEVC)," in
Applications of Digital Image Processing XXXIII, vol. 7798 of Proceedings of SPIE, August 2010.

[5] K. Ugur, K. Andersson, A. Fuldseth et al., "High performance, low complexity video coding and the emerging hevc standard," IEEE Transactions on Circuits and Systems for Video Technology, vol. 20, no. 12, pp. 1688-1697, 2010.

[6] C. C. Ju, Y. C. Chang, C. Y. Cheng et al., "A full-HD 60fps AVS/H.264/VC-1/MPEG-2 video decoder for digital home applications," in International Symposium on VLSI Design, Automation and Test (VLSI-DAT'11), pp. 117-120, April 2011.

[7] Joint Collaborative Team - Video Coding, CE10: Core transform design for HEVC, JCTVC-G495, Geneva, Switzerland, 2011.

[8] S. Lee and K. Cho, "Architecture of transform circuit for video decoder supporting multiple standards," Electronics Letters, vol. 44, no. 4, pp. 274-276, 2008.

[9] S. Kim, H. Chang, S. Lee, and K. Cho, "VLSI design to unify IDCT and IQ circuit for multistandard video decoder," in 12th International Symposium on Integrated Circuits (ISIC '09), pp. 328-331, December 2009.

[10] H. Qi, Q. Huang, and W. Gao, "A low-cost very large scale integration architecture for multistandard inverse transform," IEEE Transactions on Circuits and Systems II, vol. 57, no. 7, pp. 551-555, 2010.

[11] S. Lee and K. Cho, "Circuit implementation for transform and quantization operations of H.264/MPEG-4/VC-1 video decoder," in International Conference on Design and Technology of Integrated Systems in Nanoscale Era (DTIS '07), pp. 102-107, September 2007.

[12] K. A. Wahid, M. Martuza, M. Das, and C. McCrosky, "Efficient hardware implementation of $8 \times 8$ integer cosine transforms for multiple video codecs," Journal of Real-Time Image Processing. In press.

[13] G. Liu, "An area-efficient IDCT architecture for multiple video standards," in 2nd International Conference on Information Science and Engineering (ICISE '10), pp. 3518-3522, December 2010.

[14] C. P. Fan and G. A. Su, "Efficient low-cost sharing design of fast 1-D inverse integer transform algorithms for H.264/AVC and VC-1," IEEE Signal Processing Letters, vol. 15, pp. 926-929, 2008.

[15] C. Fan and G. Su, "Fast algorithm and low-cost hardwaresharing design of multiple integer transforms for VC-1," IEEE Transactions on Circuits and Systems II, vol. 56, pp. 788-792, 2009.

[16] D. Zhou, Z. You, J. Zhu et al., “A 1080p@60fps multi-standard video decoder chip designed for power and cost efficiency in a system perspective," in Symposium on VLSI Circuits, pp. 262263, June 2009.

[17] C. P. Fan and Y. L. Lin, "Implementations of low-cost hardware sharing architectures for fast $8 \times 8$ and $4 \times 4$ integer transforms in H.264/AVC," IEICE Transactions on Fundamentals of Electronics, Communications and Computer Sciences, vol. 90, no. 2, pp. 511-516, 2007.

[18] Y. C. Chao, S. T. Wei, C. H. Kao, B. D. Liu, and J. F. Yang, "An efficient architecture of multiple $8 \times 8$ transforms for H.264/AVC and VC-1 decoders," in 1st International Conference on Green Circuits and Systems (ICGCS '10), pp. 595-598, June 2010.

[19] Y. Li, Y. He, and S. Mei, "A highly parallel joint VLSI architecture for transforms in H.264/AVC," Journal of Signal Processing Systems, vol. 50, no. 1, pp. 19-32, 2008. 

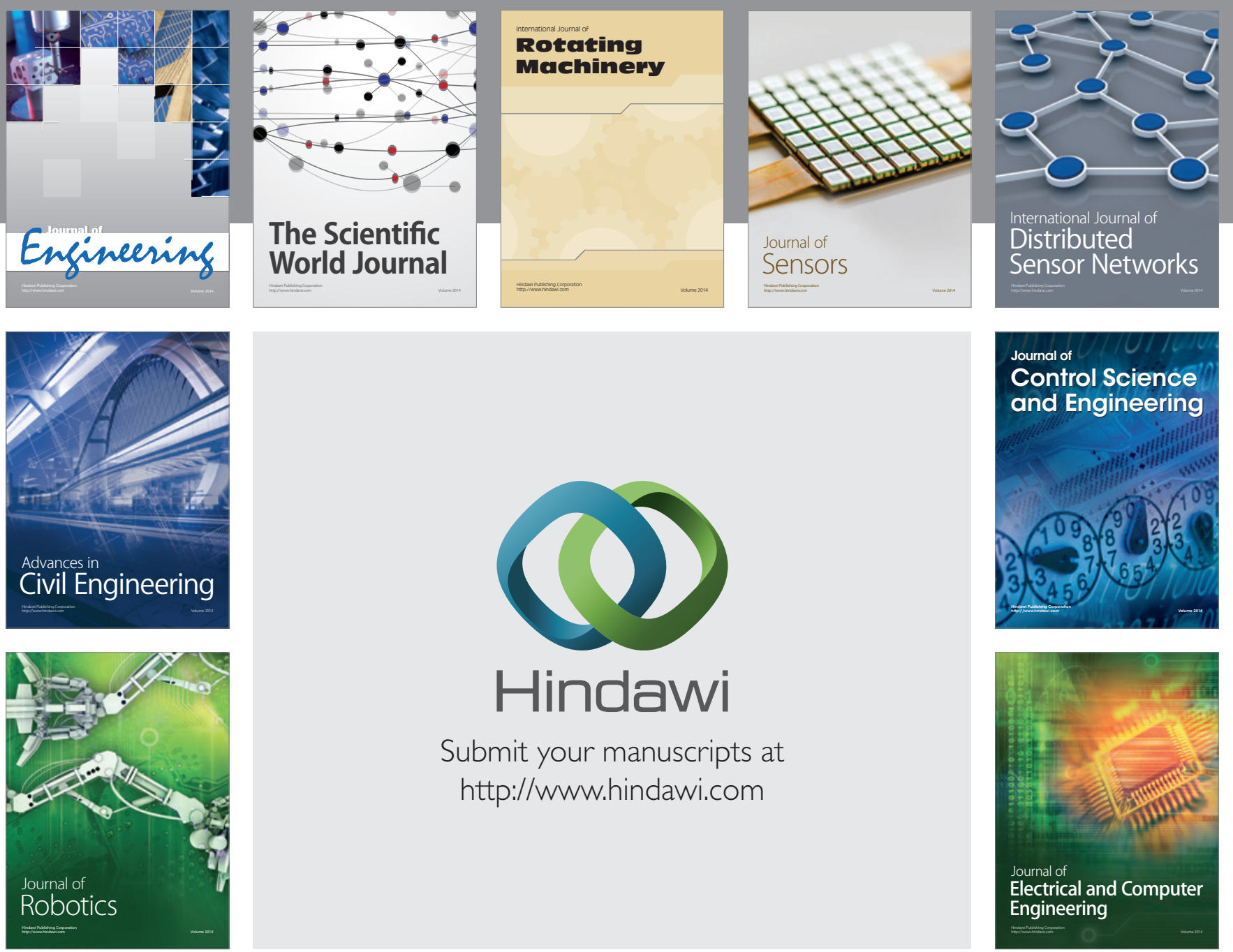

Submit your manuscripts at

http://www.hindawi.com
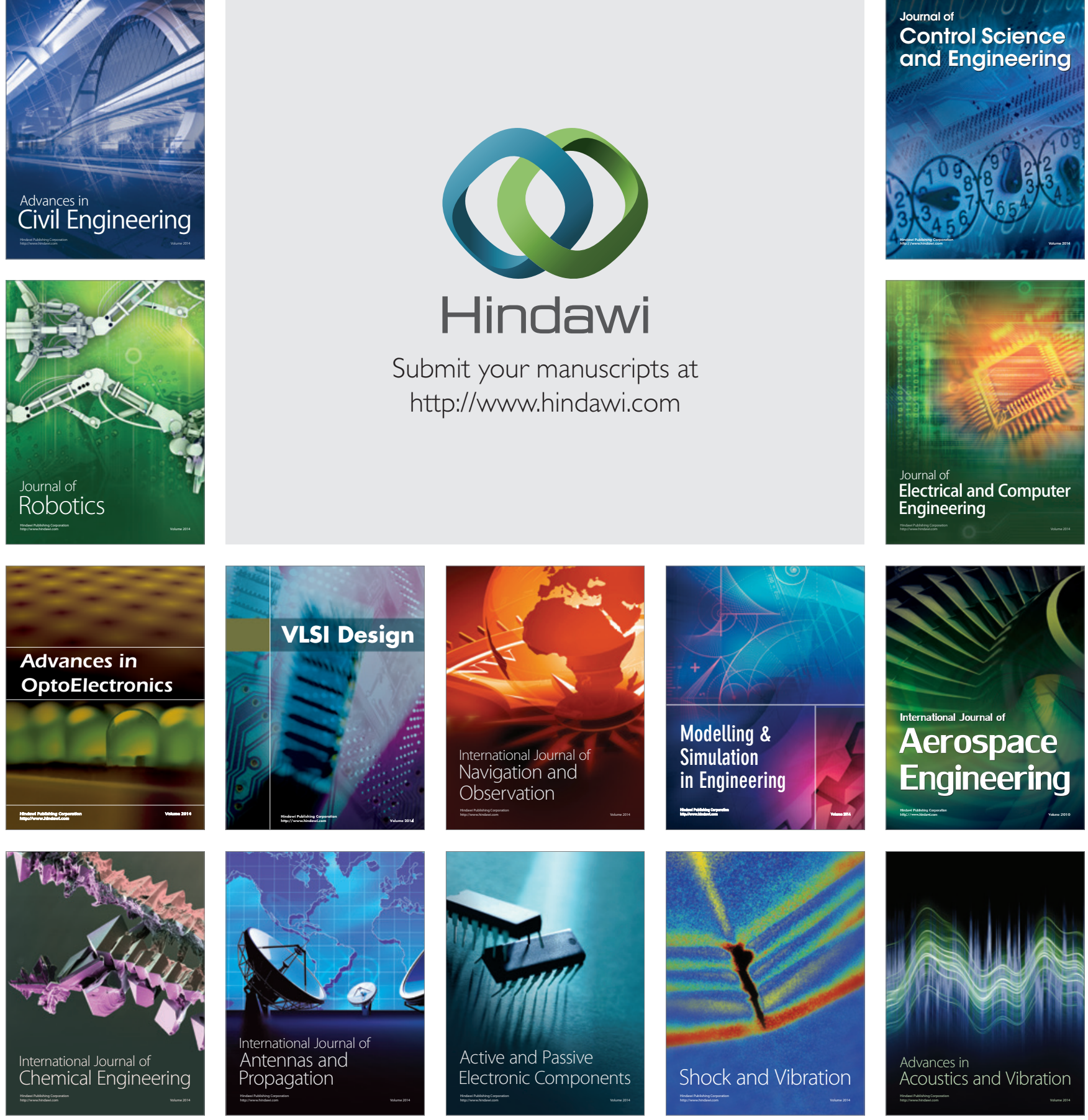